\title{
Root exudates drive the soil-borne legacy of aboveground pathogen infection
}

\author{
Jun Yuan', Jun Zhao², Tao Wen', Mengli Zhao' ${ }^{1}$, Rong Li', Pim Goossens ${ }^{3}$, Qiwei Huang ${ }^{1}$, Yang Bai ${ }^{4}$, \\ Jorge M. Vivanco ${ }^{5}$, George A. Kowalchuk ${ }^{6}$, Roeland L. Berendsen ${ }^{3}$ and Qirong Shen ${ }^{1 *}$
}

\begin{abstract}
Background: Plants are capable of building up beneficial rhizosphere communities as is evidenced by diseasesuppressive soils. However, it is not known how and why soil bacterial communities are impacted by plant exposure to foliar pathogens and if such responses might improve plant performance in the presence of the pathogen. Here, we conditioned soil by growing multiple generations (five) of Arabidopsis thaliana inoculated aboveground with Pseudomonas syringae pv tomato (Pst) in the same soil. We then examined rhizosphere communities and plant performance in a subsequent generation (sixth) grown in pathogen-conditioned versus control-conditioned soil. Moreover, we assessed the role of altered root exudation profiles in shaping the root microbiome of infected plants.

Results: Plants grown in conditioned soil showed increased levels of jasmonic acid and improved disease resistance. Illumina Miseq $16 \mathrm{~S}$ rRNA gene tag sequencing revealed that both rhizosphere and bulk soil bacterial communities were altered by Pst infection. Infected plants exhibited significantly higher exudation of amino acids, nucleotides, and long-chain organic acids (LCOAs) (C > 6) and lower exudation levels for sugars, alcohols, and short-chain organic acids (SCOAs) (C $\leq 6)$. Interestingly, addition of exogenous amino acids and LCOA also elicited a disease-suppressive response.

Conclusion: Collectively, our data suggest that plants can recruit beneficial rhizosphere communities via modification of plant exudation patterns in response to exposure to aboveground pathogens to the benefit of subsequent plant generations.
\end{abstract}

Keywords: Soil-borne legacy, Foliar pathogen, Microbiome, Disease-suppressive soil, Root exudates

\section{Background}

Plants are continually under attack from a variety of microbial pathogens that cause disease. However, some soils have the capacity to suppress plant disease even in the presence of a virulent pathogen and under climatic conditions that are favorable for disease development [1, 2]. Such naturally disease-suppressive soils have been reported for diseases across a diverse range of agricultural crops, such as Take-all and Rhizoctonia bare patch disease on wheat [1], potato common scab [3], Fusarium wilt on strawberry and vanilla $[4,5]$, and Rhizoctonia solani on sugar beet [6]. In some disease-suppressive

\footnotetext{
* Correspondence: shenqirong@njau.edu.cn

1Jiangsu Provincial Key Lab for Organic Solid Waste Utilization; National Engineering Research Center for Organic-based Fertilizers; Jiangsu Collaborative Innovation Center for Solid Organic Waste Resource Utilization, Nanjing Agricultural University, Nanjing 210095, China

Full list of author information is available at the end of the article
}

soils, this suppressiveness is related to the abundance of specific microbes in the soil $[4,6]$. In high abundance, beneficial microbes can directly inhibit pathogens by producing antimicrobial compounds. However, beneficial microbes can also inhibit pathogens indirectly by stimulating the plant's immune system, a phenomenon called induced systemic resistance (ISR) [7]. In order for disease-suppressive communities to develop, successive cropping cycles of the same plant species need be grown in the presence of a severe disease outbreak. This observation has led to the hypothesis that, upon attack, plants enrich and sustain specific beneficial microbes that come to their aid $[1,8,9]$. Some evidence for this "cry for help" hypothesis is beginning to accumulate. In the case of wheat and pepper, defense activation has been demonstrated to stem from plant-mediated changes in rhizosphere microbial communities $[10,11]$. In the model plant Arabidopsis thaliana (hereafter referred to as

(c) The Author(s). 2018 Open Access This article is distributed under the terms of the Creative Commons Attribution 4.0 International License (http://creativecommons.org/licenses/by/4.0/), which permits unrestricted use, distribution, and 
Arabidopsis), the infection of leaves by Pseudomonas syringae pathovar tomato DC3000 (hereafter referred to as Pst) has been shown to induce the secretion of malic acid by roots, which led to the promotion of the ISR-inducing Bacillus subtilis strain FB17 on gnotobiotic roots of infected plants. Moreover, it was shown recently that also in natural soils, Arabidopsis plants can promote a select group of beneficial microbes in the rhizosphere [12]. Upon foliar defense activation by the downy mildew Hyaloperonospora arabidopsidis, a beneficial consortium including a Xanthomonas sp., a Stenotrophomonas sp., and Microbacterium sp. populations was strongly promoted in the rhizosphere. Furthermore, upon isolation, these strains could together induce resistance to down mildew when inoculated back to Arabidopsis. Moreover, downy mildew infection in a first population of plants increased the resistance of a second population of plants growing in the same soil. Together, these results indicate that plants can recruit beneficial microbes upon attack to generate a soil memory or "soil-borne legacy" that better prepares the next generation of plants to avoid harmful effects of the pathogen [12-15]. In this process, root exudates and other root-derived molecules are believed to play a role [12, 13, 16-18], although direct evidence supporting this hypothesis is generally lacking. In this study, we examined the role of root exudates in the establishment of soil-borne legacies following foliar pathogen attack. We conditioned soils by growing five successive generations of Arabidopsis in the same soil. In each generation, plants were either infected or not infected with Pst. In this way, we generated pathogen-conditioned soils as well as control-conditioned soils. We subsequently examined plant growth and hormone production in the following (sixth) generation in the absence of the pathogen. We also tracked the soil bacterial community in the bulk as well as the rhizosphere soil by high-throughput $16 \mathrm{~S}$ rRNA gene tag sequencing. Concurrently, we identified compounds that were differentially exuded by infected versus uninfected plants and tested these compounds for their ability to promote disease suppressiveness in soil. Finally, we examined whether these compounds affect plant resistance directly or indirectly through their effects on the microbiome. By combining these complementary lines of investigation (Additional file 1: Figure $\mathrm{S} 1$ ), we were able to examine how changing exudation patterns can act as a mechanism by which plants can build their soil-borne legacy to the benefit of future plant generations.

\section{Methods}

\section{Development of pathogen-conditioned soils}

The soil used in this experiment was collected in July 2014 from a site near the Michigan Extension Station
(Benton Harbor: N 4205'34", W 86'21'19") where Arabidopsis thaliana genotype Pna-10 has grown naturally for more than a decade. Soils from this site have been used in several previous studies [16, 19, 20]. The collected soil was transported to the laboratory in airtight coolers and stored in a cold room $\left(4{ }^{\circ} \mathrm{C}\right)$ until use. Before the start of the experiment, all soils were dried at room temperature, pooled, and thoroughly sieved to remove roots and other plant tissue.

To condition soils for five generations, Arabidopsis thaliana accession Col-0 plants were first sown on Murashige and Skoog [21] ager plates supplemented with $1 \%$ sucrose stratified for 2 days at $4{ }^{\circ} \mathrm{C}$ and allowed to germinate and grow in a climate chamber $\left(25 \pm 2{ }^{\circ} \mathrm{C}, 16 \mathrm{~h}\right.$ light/ $8 \mathrm{~h}$ dark, light intensity $45 \mu \mathrm{mol} \mathrm{m} \mathrm{s}^{-1}$; these conditions were used for plant growth throughout this study). Four 7-day-old seedlings were transferred to each of 108 pots ( 3 pots per set, 18 replicate sets for each treatment). Each pot contained $50 \mathrm{~g}$ of soil. The plants in half of the pots (54 pots) were inoculated with Pst at 14 days post-transplantation. Four true leaves of each plant (16 leaves per pot) were punctured using a syringe needle and $1 \mu \mathrm{L}$ of a Pst suspension $\left(10^{7} \mathrm{CFU} / \mathrm{mL}\right.$, Pst was pre-cultured overnight in nutrient broth at $37{ }^{\circ} \mathrm{C}$ with $170 \mathrm{rpm}$ shaking and cell density adjusted by addition of sterilized water) was added to the wound. Special care was taken to avoid contamination of the soil with the Pst inoculum. Sterilized water was added to the punctures in control-treated plants (the other 54 pots). After the Pst suspensions had air-dried on the leaves, all pots were randomized, placed back into the growth chamber, and covered to maintain high humidity. Pst infections were allowed to develop for 14 days, at which time wounded leaves became diseased as evidenced by a clearly visible necrotic area. Subsequently, the aboveground plant parts, but not the roots, were removed, and the pots containing the soil were airdried for 1 week. Subsequently, $5 \mathrm{~mL}$ of MS medium was added to each pot and new 7-day-old seedlings were transplanted to the pots for the next generation. This process was repeated for a total of five consecutive plant generations with the same batch of seeds (Additional file 1: Figure S1).

\section{Assessment of disease resistance on conditioned soils}

One 7-day-old Arabidopsis seedling was transferred to the center of each of the 27 pathogen-conditioned and 27 control-conditioned pots (conditioned as described above) and incubated in a climate chamber. Fourteen days after transplantation, all plants were inoculated with Pst as described above. Seven days after inoculation, the number of necrotic leaves as a percentage of the total number of inoculated leaves was recorded to determine the disease incidence. 


\section{Microbiome sample collection and determination of phytohormone levels in the absence of the pathogen}

The remaining conditioned soils (27 pots for each of the two treatments) were used to assess the effect of conditioning on phytohormone levels and microbiome composition in the absence of the pathogen. Again, one 7-day-old Arabidopsis seedling was transferred to the center of each conditioned pot. After 28 days of growth, aboveground plant biomass was harvested, weighed, and analyzed for phytohormone content. Rhizosphere soils were collected as described previously [19]. Briefly, roots were gently removed from soil together with the tightly adhering soil. The bulk soil was also collected after the rhizosphere soil collection (remaining soil). Within each treatment, samples from three randomly selected pots were pooled, thereby yielding nine composite samples for rhizosphere and bulk soils for both the pathogenconditioned and control-conditioned soils. All samples were immediately stored at $-80{ }^{\circ} \mathrm{C}$ prior to subsequent DNA analysis.

Phytohormones in shoot tissue were extracted from $100 \mathrm{mg}$ pre-ground aboveground fresh tissue in $1 \mathrm{~mL}$ cold extraction solvent (20:79:1, methanol: isopropanol: acetic acid, v:v:v). Ten microliters of internal standard solution (jasmonic acid (JA): $10 \mathrm{ng} / \mathrm{mL}$, salicylic acid (SA) $60 \mathrm{ng} / \mathrm{mL}$, and abscisic acid (ABA) $10 \mathrm{ng} / \mathrm{mL}$ ) was added to each sample. The extraction process and LC-MS/MS analysis were performed as previously described [22]. Specifically, tandem mass spectrometry coupled to liquid chromatography was performed on a Waters Xevo TQ-S triple quadruple mass spectrometer coupled to a Waters M-class Acquity UPLC system. Optimization of parent ion, cone voltage, collision energies, and fragment ions was done via direct infusion of phytohormones into the mass spectrometer for selected reaction transitions. LC-MS/MS buffers were as follows: buffer A was water with $0.1 \%$ formic acid and buffer B consisted of acetonitrile with $0.1 \%$ formic acid. Buffers were added in a gradient as follows: $10 \%$ buffer B was used to start, at 8 min $97 \%$ buffer B, and at 9.5 min $10 \%$ buffer B. We used a Waters $3 \mu \mathrm{M}$ Atlantis dC18 $(300 \mu \mathrm{M} \times 150 \mathrm{~mm})$ column set to a flow rate of $11.5 \mu \mathrm{L} / \mathrm{min}$ with column temperature held at $40{ }^{\circ} \mathrm{C}$. Injection volume was $1 \mu \mathrm{L}$ and autosampler temperature was set at $4{ }^{\circ} \mathrm{C}$. Waters TargetLynx software was used for data analysis and hormone quantification. Quantification of phytohormones was done with the following formula: analyte peak area $\times$ (internal standard concentration/internal standard peak area). By utilizing polarity switching, all samples were analyzed via LC-MS in a single run with dwell times of $10 \mathrm{~ms}$. In positive ion mode, capillary voltage was $3.2 \mathrm{kV}$, while in negative ion mode, it was - 2.2 kV. Nebulizer gas flow was 7.0 bar, cone gas flow was $150 \mathrm{~L} / \mathrm{h}$, desolvation temperature was $225^{\circ} \mathrm{C}$, and desolvation gas flow was $825 \mathrm{~L} / \mathrm{h}$. Argon was used as collision gas at a flow rate of $0.2 \mathrm{~mL} / \mathrm{min}$.

\section{PCR amplification and sequencing}

Total DNA was extracted from a total of 18 rhizosphere samples (9 for each treatment) and 18 bulk soil samples. Extractions were carried out on $500 \mathrm{mg}$ of soil (wet weight) using the Power Soil DNA Isolation kit (Mo Bio Laboratories Inc., Carlsbad, CA, USA), according to the manufacturer's instructions. DNA was extracted from two technical replicates per sample to minimize the DNA extraction bias. Samples were stored at $-20{ }^{\circ} \mathrm{C}$, and technical replicates were pooled before performing polymerase chain reaction. The DNA quality was assessed according to the 260/280-nm and 260/230-nm absorbance ratios using a NanoDrop ND-2000 spectrophotometer (NanoDrop, ND2000, Thermo Scientific, 111 Wilmington, DE). The concentration of extracted DNA was between 40 and $60 \mathrm{ng} / \mu \mathrm{L}$. Bacterial 16S rRNA gene fragments were amplified from the extracted DNA using primers 341F (CCTAYGGGRBGCASCAG) and 806R (GGACTACHVGGGTWTCTAAT) and the following PCR conditions: $30 \mathrm{~s}$ at $95{ }^{\circ} \mathrm{C}, 30 \mathrm{~s}$ at $59{ }^{\circ} \mathrm{C}$, and $30 \mathrm{~s}$ at $72{ }^{\circ} \mathrm{C}$ for 30 cycles. PCRs were performed in a total volume of $25 \mu \mathrm{L}$ with $9.75 \mu \mathrm{L}$ water, $5 \mu \mathrm{L} 5 \times$ PCR buffer, $5 \mu \mathrm{L} 5 \times$ Q5 GC high enhancer, $2 \mu \mathrm{L}$ deoxynucleoside triphosphates (dNTPs), $1 \mu \mathrm{L}$ of each primer, $0.25 \mu \mathrm{L}$ and $5 \mathrm{U} / \mu \mathrm{L}$ of $\mathrm{Q} 5$ polymerase, and $1 \mu \mathrm{L}$ of extracted DNA [23]. After PCR amplification, bands were excised and purified from 1.5\% agarose gels using the MinElute PCR Purification Kit (Qiagen, Germany) and the QiagenQIAquick Gel Extraction kit (Qiagen, Germany). Amplicons were subjected to paired-end sequencing on the Illumina MiSeq sequencing platform using PE250 chemical at the Genomics Core of Michigan State University. After assembly, we got $\sim 480 \mathrm{bp}$ reads covering the $\mathrm{V} 3-\mathrm{V} 4$ region of the bacterial $16 \mathrm{~S}$ rRNA.

\section{Amplicon sequence processing and analysis}

Amplicon sequences were analyzed using the Qiime2 environment (version 2017.12, https://qiime2.org/). Initial sequence quality was assessed using the "Demux" plugin. Due to low sequence quality at the 3 '-ends of the reads, joining paired-ends resulted in unreliable reads and greatly reduced the amount of paired-end reads that passed quality control. We therefore only used the forward reads (containing the relatively short but highly discriminating V3 region) and employed the DADA2 pipeline [24]. Sequences were truncated at base 140 and trimmed until base 17. This resulted in relatively short reads $(\sim 120 \mathrm{bp})$ of high quality from which actual sequence variants (ASVs) were identified. The 
DADA2 pipeline then produced an ASV count table containing 1.35 million usable reads and $\sim 5000$ ASVs. In some cases, we observed ASVs that were highly abundant in one sample but absent from the rest of the dataset. These were judged to likely be from chimeric sequences that were not filtered out in the DADA2 pipeline (as a consequence of using only the forward reads) and excluded from further analysis. The resulting final ASV table contained $\sim 900,000$ high-quality reads belonging to $\sim 2000$ ASVs. Taxonomic assignment of ASVs was performed using the VSEARCH consensus taxonomy classifier implemented in Qiime2 and the SILVA 16S rRNA database [25]. Statistical analyses of the $16 \mathrm{~S}$ rRNA microbiome sequencing data was performed using the Qiime2 environment (version 2017.12) and in the R environment (R 2017, https://www.r-project.org version 3.4.3). $\beta$-diversity (PCoA based on BrayCurtis dissimilarities) was calculated using the "phyloseq" package (version 1.22.3) [26]. Statistical significance of the $\beta$-diversity between treatments was calculated through analysis of similarity (ANOSIM), as implemented in the Qiime2 environment. Graphs of the microbiome data were created using the "ggplot2" package (version 2.2.1). The code used in this process was listed in Additional file 1.

\section{Pst detection in soil by PCR}

The detection of Pst was carried out using the PCR primers OWB575 (AACTGAAAAACACCTTGGGC) and OWB576 (CCTGGGTTGTTGAAGTGGTA) that target the Oprf gene of $P$. syringae [27]. PCR conditions were as follows: an initial $95{ }^{\circ} \mathrm{C}$ for $4 \mathrm{~min}$ and 30 cycles of $30 \mathrm{~s}$ at $95{ }^{\circ} \mathrm{C}, 30 \mathrm{~s}$ at $59{ }^{\circ} \mathrm{C}$, and $30 \mathrm{~s}$ at $72{ }^{\circ} \mathrm{C}$. PCRs were performed in a total volume of $25 \mu \mathrm{L}$ with $17.2 \mu \mathrm{L}$ water, $2.5 \mu \mathrm{L} 10 \times$ PCR buffer $\left(\mathrm{Mg}^{2+}\right.$ plus), $2 \mu \mathrm{L}$ deoxynucleoside triphosphates (dNTPs), $1 \mu \mathrm{L}$ of each primer, $0.3 \mu \mathrm{L}$ Taq polymerase (TaKaRa Biotechnology Co., Ltd), and $1 \mu \mathrm{L}$ of extracted DNA. Water was used as the negative control, and genomic DNA extract from Pst was the positive control. The PCR products were visualized by electrophoresis through a $1 \%$ agarose gel.

\section{Soil chemical analyses}

The soil chemical analyses were conducted on $5 \mathrm{~g}$ of bulk soil collected at the sixth generation of plant growth after the aboveground plant parts were removed. Both conditioned and control soil samples were used to analyze available phosphorus (AP), available potassium (AK), nitrate $\left(\mathrm{NO}_{3}{ }^{-}\right)$, ammonia $\left(\mathrm{NH}_{4}{ }^{+}\right)$, and $\mathrm{pH}$ [28]. All analyses were performed at the Soil, Water and Plant Testing Laboratory at Colorado State University.

\section{Root exudate collection and GC-MS analysis}

For root exudate collection, surface-sterilized Arabidopsis (Col-0) seeds were placed on MS agar-solidified medium ( $1 \%$ agar) amended with $3 \%$ sucrose, stratified for 2 days at $4{ }^{\circ} \mathrm{C}$, and allowed to germinate and grow for 14 days in a climate chamber. Plants were subsequently inoculated with Pst as described above and, after the pathogen suspension had air-dried on the leaves, plants were transferred to 6 -well plates containing water-agar medium ( $1 \%$ agar) for exudate collection. Plates were randomized during the collection period. Each treatment contained three replicate plates with six plants. Non-inoculated but wounded plants were used as control.

Plates were covered and incubated in the climate chamber for 3 days. After 3 days of collection, the water-agar medium of the 6 wells in one plate were pooled as one sample and lyophilized for further extraction. Root exudates were extracted from the lyophilized agar medium with $80 \%$ methanol for further extraction and GC-MS analyses were performed as previously described [16]. Briefly, extracts were dried under nitrogen gas and then methoximated and trimethylsilylated. The derivatives were analyzed by an Agilent 6890 gas chromatograph (Santa Clara, CA) containing a 30-m-long, $0.25-\mathrm{mm}$ inner diameter rtx5Sil-MS column with an additional 10-m integrated guard column. Metabolites were detected using the BinBase algorithm [29] and identified by comparing the retention index and mass spectrum of each analyte against the Fiehn mass spectral library from the West Coast Metabolomics Center, University of California Davis. The experiment was repeated and compounds were analyzed using the same method at BIOTREE technology Co. Ltd. in Shanghai, China, and similar results were obtained.

\section{Impacts of root exudate classes on soil microbiome feedbacks to plant defense}

To examine the effect of root exudates secreted upon aboveground Pst infection on soil suppressiveness, we selected four categories of differentially secreted exudates: long-chain organic acids (LCOAs), amino acids (AAs), short-chain organic acids (SCOAs), and sugars. For each exudate category, representative compounds were selected based on their altered abundance in root exudates after Pst infection. For the AA, SCOA, and sugar category, watery solutions were prepared containing each of the selected compounds in equal dosage and to a final total concentration of $10 \mathrm{mM}$. For LCOAs, the total concentration was $10 \mu \mathrm{M}$ due to their lower solubility. The LCOA solution contained $2.0 \mu \mathrm{M}$ pentadecanoic acid, $2.0 \mu \mathrm{M}$ hexadecanoic acid, $2.0 \mu \mathrm{M}$ palmitoleic acid, $2.0 \mu \mathrm{M}$ octadecanoic acid, and $2.0 \mu \mathrm{M}$ arachidic acid. The AA solution contained $1.67 \mathrm{mM}$ isoleucine, 
$1.67 \mathrm{mM}$ leucine, $1.67 \mathrm{mM}$ methionine, $1.67 \mathrm{mM}$ proline, $1.67 \mathrm{mM}$ tryptophan, and $1.67 \mathrm{mM}$ ornithine. The SCOA solution contained $2.5 \mathrm{mM}$ citric acid, $2.5 \mathrm{mM}$ aconitic acid, $2.5 \mathrm{mM}$ succinic acid, and $2.5 \mathrm{mM}$ malic acid. The sugar solution contained $1.67 \mathrm{mM}$ maltose, $1.67 \mathrm{mM}$ ribose, $1.67 \mathrm{mM}$ glucose, $1.67 \mathrm{mM}$ sucrose, $1.67 \mathrm{mM}$ fructose, and $1.67 \mathrm{mM}$ xylose.

Fifteen grams of soil was placed into each well of a 6-well plate. Plates were pre-incubated in a growth chamber at $30{ }^{\circ} \mathrm{C}$ for 1 week to allow the soil microbiome to acclimatize and to remove seedlings from the naturally occurring seedbank. Each well then received $1.5 \mathrm{~mL}$ of exudate compound solution twice a week for $81 / 2$ weeks (17 total applications) in a growth chamber at $30{ }^{\circ} \mathrm{C}$. We applied three treatments: (1) $\mathrm{L}+\mathrm{A}$, equal volumes of the LCOA and AA solutions (representing pathogen-induced root exudates); (2) $\mathrm{S}+\mathrm{S}$, equal volumes of SCOA and sugar solutions (representing pathogen-repressed root exudates); and (3) water control. Each treatment consisted of 18 replicates divided over 3 plates. All plates were randomly placed during the incubation period.

To examine the effect of exudate compound classes on the development of soil suppressiveness, soil slurries were prepared and filter-sterilized as described previously [22]. Briefly, $5 \mathrm{~g}$ of each soil treatment was mixed with $50 \mathrm{~mL}$ autoclaved water on an orbital shaker for $1 \mathrm{~h}$. After settling for an additional hour, soil slurry was obtained from the supernatant through filter paper and filtered slurry were filter-sterilized using a $0.22-\mu \mathrm{m}$ filter polytetrafluoroethylene (PTFE) membrane. Slurries were prepared of the LA, SS, control-treated soils, and mixtures of LA and SS treated soils at ratios of 9:1, 5:5, and 1:9 $(v / v)$. Seven-day-old Arabidopsis seedlings (prepared as described above) were transplanted to an autoclaved mixture of vermiculite and sand (volume: volume $=1: 1$ ) in 6-well plates to which $2 \mathrm{~mL}$ of soil slurry, filter-sterilized soil slurry, or autoclaved water was added. Five milliliters of MS medium was added to supply plants with the necessary nutrition during plant growth period. After 2 weeks, the Pst strain was inoculated onto the Arabidopsis leaves as described above. Disease incidence was determined 7 days after infection.

\section{Statistical methods}

Statistically significant differences $(p<0.05)$ in disease incidence, plant biomass, phytohormones, root exudate compound abundance, and soil properties between controls and treatments were evaluated by Student's $t$ test or ANOVA using SPSS. The differences in bacterial community composition or in the root exudate composition among treatments were tested using PERMANOVA (Adonis, transformed data by Bray-Curtis, permutation $=999)$, implemented in $\mathrm{R}$ version 3.4.3.
The DESeq function of the "DESeq2" package (version 1.18.1) [30] was employed to test for differentially abundant ASVs in pathogen- and control-conditioned bulk soil and rhizosphere samples. Statistical significance was based on $p$ value $<0.05$ (with FDR $<5 \%$ under the Benjamini-Hochberg correction).

\section{Results and discussion}

Effect of pathogen-conditioned soil on plant performance Soils were conditioned by five succeeding generations of Arabidopsis plants of which the leaves were either infected by Pst (pathogen-conditioned soil) or subjected to a mock treatment (control soil). When a sixth generation of plants was planted on these conditioned soils and confronted with Pst, plants grown in pathogen-conditioned soils developed significantly $(p<0.05)$ reduced disease symptoms as compared to plants grown in control-conditioned soil (Fig. 1a). This supports previous findings that aboveground pathogen infection of a population of plants leads to a soil-borne legacy that induces resistance in a following population of plants growing in the same soil $[12,14]$.

In the absence of pathogen, plants grown in pathogenconditioned soil exhibited significantly $(p<0.05)$ reduced fresh biomass compared to plants grown in the control soil (Fig. 1b). It is known that the activation of defenses comes at a cost for plant performance in the absence of the pathogen [31]. It is thus likely that the observed growth depression on pathogen-conditioned soil was the result of a redirection of plant metabolism toward defense at a cost of growth [32]. It should be noted that we did not detect any significant differences $(p>0.05, t$ test $)$ in $\mathrm{pH}$ and nutrient content $\left(\mathrm{NH}_{4}{ }^{+}, \mathrm{NO}_{3}{ }^{-}, \mathrm{AP}\right.$, and $\left.\mathrm{AK}\right)$ between the pathogen-conditioned and control soils (Additional file 1: Table S1). Thus, although we cannot rule out that there are other chemical differences between the soils of the two treatments, our data suggests that nutrient availabilities were not driving differences in plant growth in response to soil conditioning.

We then compared levels of the defense-regulating phytohormones jasmonic acid (JA), salicylic acid (SA), and abscisic acid (ABA) in unchallenged plants growing on differently pre-conditioned soils (Fig. 1c). JA levels were significantly higher $(p<0.05)$ in plants grown in pathogen-conditioned soil, while SA concentrations were significantly lower $(p<0.05)$. No significant differences were observed for abscisic acid (ABA). This indicates that the soil-borne legacy brought about by previous generations of pathogen-challenged plants induced production of JA in a sixth generation of unchallenged plants leading to defense activation and increased resistance. This suggests that the higher concentration of jasmonic acid induced by pathogen-conditioned soils resulted in reduced SA levels, as the SA- and JA-signaling 


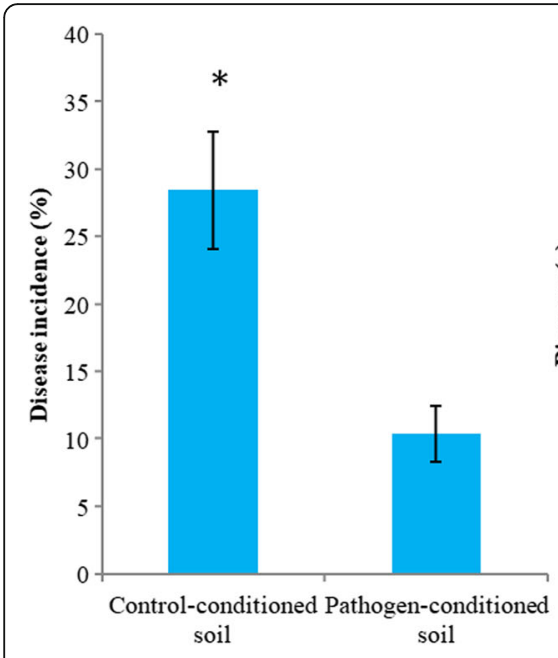

A

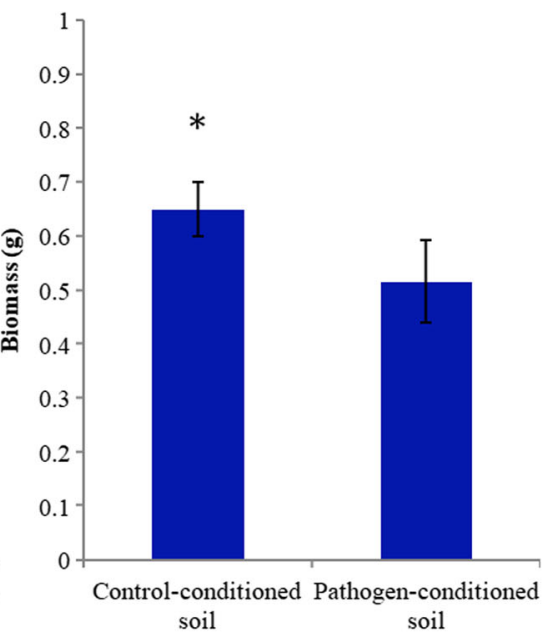

B

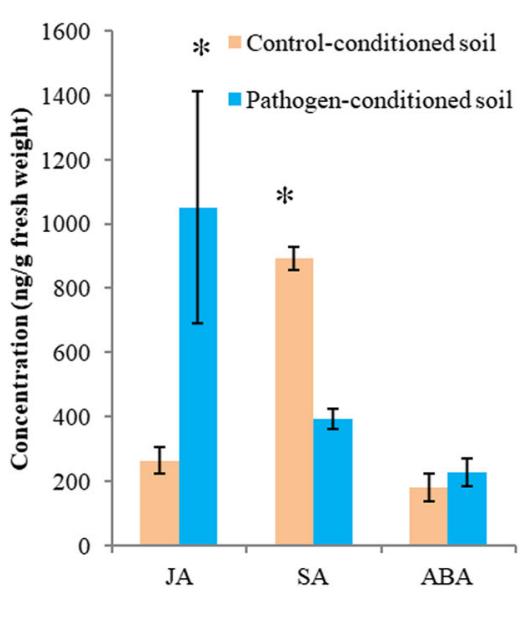

C

Fig. 1 a Disease incidence after Pst inoculation of a sixth generation of Arabidopsis plants growing on control or pathogen-conditioned soils. The asterisk indicates statistically significant differences as determined with a Student's $t$ test $(p<0.05)$ between treatments. Bars represent the average of three replicates and error bars show standard deviations. b Fresh shoot weight of unchallenged Arabidopsis plants growing on control or pathogenconditioned soil. c Concentrations of the phytohormones jasmonic acid (JA), salicylic acid (SA), and abscisic acid (ABA) in shoots of unchallenged Arabidopsis plants growing on control soil or pathogen-conditioned soil. The asterisk indicates statistically significant differences between treatments as determined with a Student's $t$ test, $(p<0.05)$. Bars represent the average of three replicates and error bars show standard deviations

pathways are known to antagonize each other [33]. Although ABA is known to fine-tune defense responses, it is mostly involved in response to abiotic stress [34-36]. It was therefore not surprising to find that biological soil conditioning had no effect on ABA levels.

It is important to note that the increased resistance in pathogen-conditioned soil was most likely not the result of the presence of Pst in the soil. The pathogens as only introduced onto the leaves and the aboveground plant parts were removed after 14 days, thereby minimizing the degree to which the pathogen might enter the soil. Moreover, Pst abundance was below detection limits in soil throughout the experiment as tested by PCR (Additional file 1: Figure S2) and qPCR [37].

\section{Impacts of aboveground pathogen infection on soil bacterial communities}

We analyzed the composition of microbial communities in the bulk soil and rhizosphere of unchallenged Arabidopsis plants growing on pathogen-conditioned or control soils using $16 \mathrm{~S}$ rRNA gene amplicon sequencing. For this analysis, we collected 9 rhizospheres and 9 bulk soil samples from both pathogen-conditioned and controlconditioned soils, resulting to total of 36 microbial communities. We obtained an average read count per sample of 25,068 (standard deviation (SD) 6568). As typical of soils, bacterial communities were highly diverse as was reflected by the numbers of actual sequence variants (ASVs), generally ranged between 285 and 708 per sample with an average of 467 (SD 117). The majority of ASVs belonged to the phyla Proteobacteria (34.8\%), Acidobacteria (20.6\%), Chloroflexi (16.1\%), Actinobacteria (7.6\%), and Firmicutes (5.9\%) (Fig. 2a). Principal coordinate analysis (PCoA) based on the detected ASVs showed a clear difference in community composition between bulk and rhizosphere soils, which was statistically significant as determined through analysis of similarity (PERMANOVA) $\left(p=0.001, R^{2}=0.39\right)$ (Fig. 2b). This demonstrates a general rhizosphere effect of the plant $[38,39]$, even in soils that had been pre-conditioned by five generations of plant growth. Moreover, there was a statistically significant effect of the conditioning treatment in both the bulk soils $(p=0.001$ in PERMANOVA, Additional file 1: Figure $\mathrm{S} 3 \mathrm{~A})$ as well as the rhizosphere soils $(p=0.031$ in PERMANOVA; Additional file 1: Figure S3B). Thus, aboveground Pst infections triggered a plant-mediated shift in belowground microbial community structure. The effect of aboveground infection, however, was more pronounced in bulk soil as compared to the rhizosphere, likely because we analyzed microbial communities only for unchallenged plants. We hypothesize that the rhizosphere effect of the unchallenged plants reduced our ability to observe a clear effect of pathogen conditioning. Upon closer inspection of the pathogen-conditioned bulk soils, these were relatively enriched in populations belonging to the Firmicutes, yet relatively depleted in Proteobacteria as compared to control soils (Fig. 2a). Furthermore, we detected that two ASVs, belonging to the genera Fictibacillus and Sphingomonas, respectively, seem to drive this separation, despite the fact that these ASVs did not differ significantly 


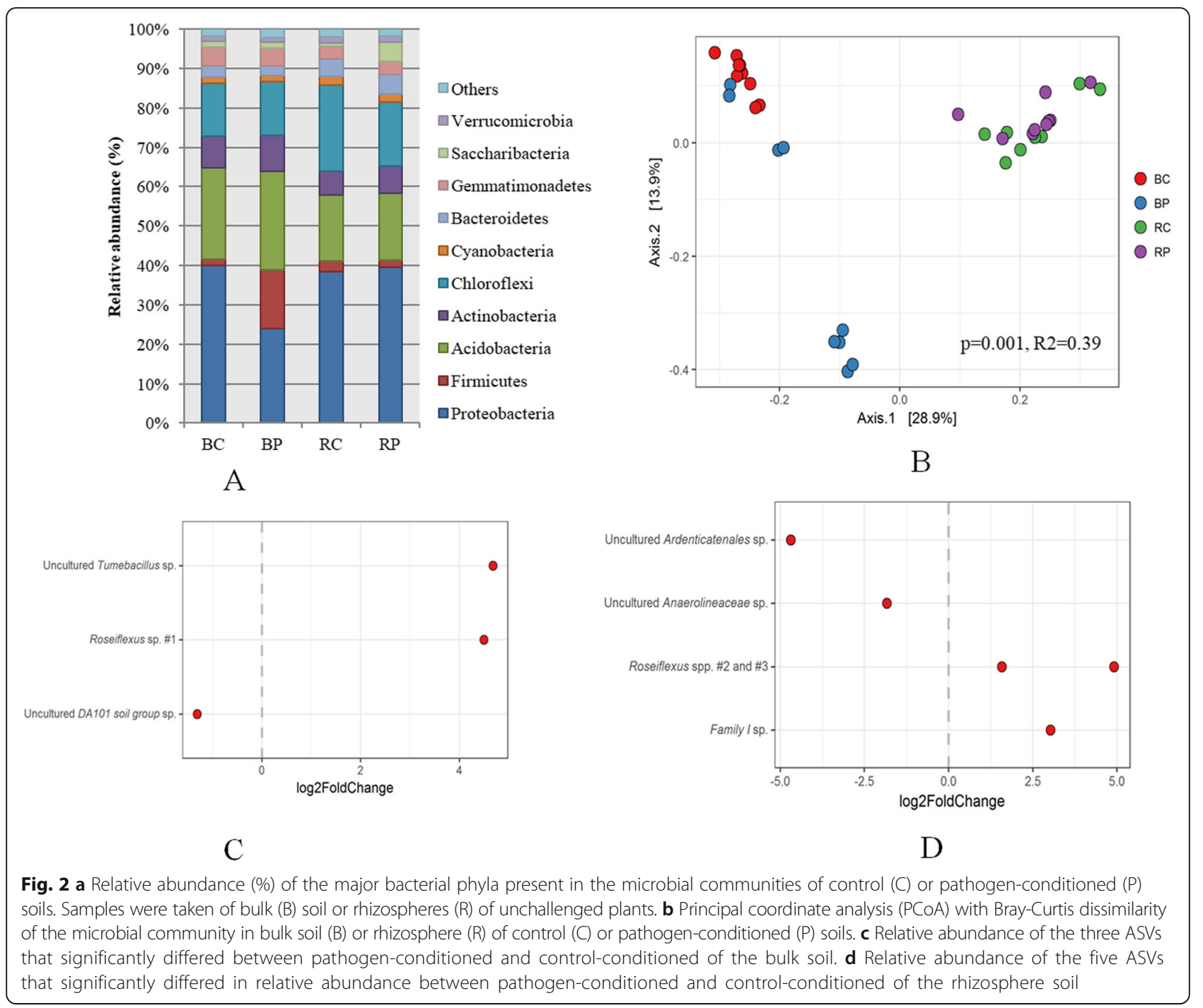

between the two treatments $(p=0.051$ and 0.859 for the Fictibacillus ASV and the Sphingomonas ASV, respectively). The relative abundance of the Fictibacillus ASV was over $20 \%$ in five-out-of-nine pathogen-conditioned bulk soil samples, while it represented $<1 \%$ of the total community in the remaining four bulk soil samples and all of the rhizosphere samples (Additional file 1: Figure S4A and Table S2). Similarly, the relative abundance of the Sphingomonas ASV was greater than $11 \%$ in all bulk soil and rhizosphere samples except these same five pathogen-conditioned bulk soil samples in which the relative abundance was below $2 \%$ (Additional file 1: Figure S4B and Table S2). In a PCoA of these communities after the exclusion of these two ASVs, the separation of these five samples is no longer apparent, although the differences between the two conditioning treatments was still statistically significant $(p=0.001$ in PERMANOVA, Additional file 1: Figure S5). The difference of average relative abundance of Firmicutes and Proteobacteria in bulk soils was also lost (Additional file 1: Figure
S4C). Previously, it was found that aboveground downy mildew infection led to a very specific recruitment of three bacterial species to the roots of Arabidopsis [12]. Our data also show that only a very select number of ASVs is significantly impacted $(p<0.05)$ by the pathogen-conditioning soil treatment as compared to the control (Fig. 2c, d). Three ASVs differed between these treatments in the bulk soil (Fig. 2c) and five ASVs were significantly affected by the treatment in the rhizosphere samples (Fig. 2d). Of the ASVs affected by the treatment, five ASVs were more abundant in pathogen-conditioned soils, whereas three ASVs were less abundant. Remarkably, three ASVs that were significantly enriched in either pathogen-conditioned bulk or pathogen-conditioned rhizosphere soils belonged to the same genus, i.e., Roseiflexus.

These results thus appear to indicate that indeed only a few microbial populations are recruited by Pst-infected plants. However, even after removal of the most differential 200 ASVs, the remaining parts of the communities 


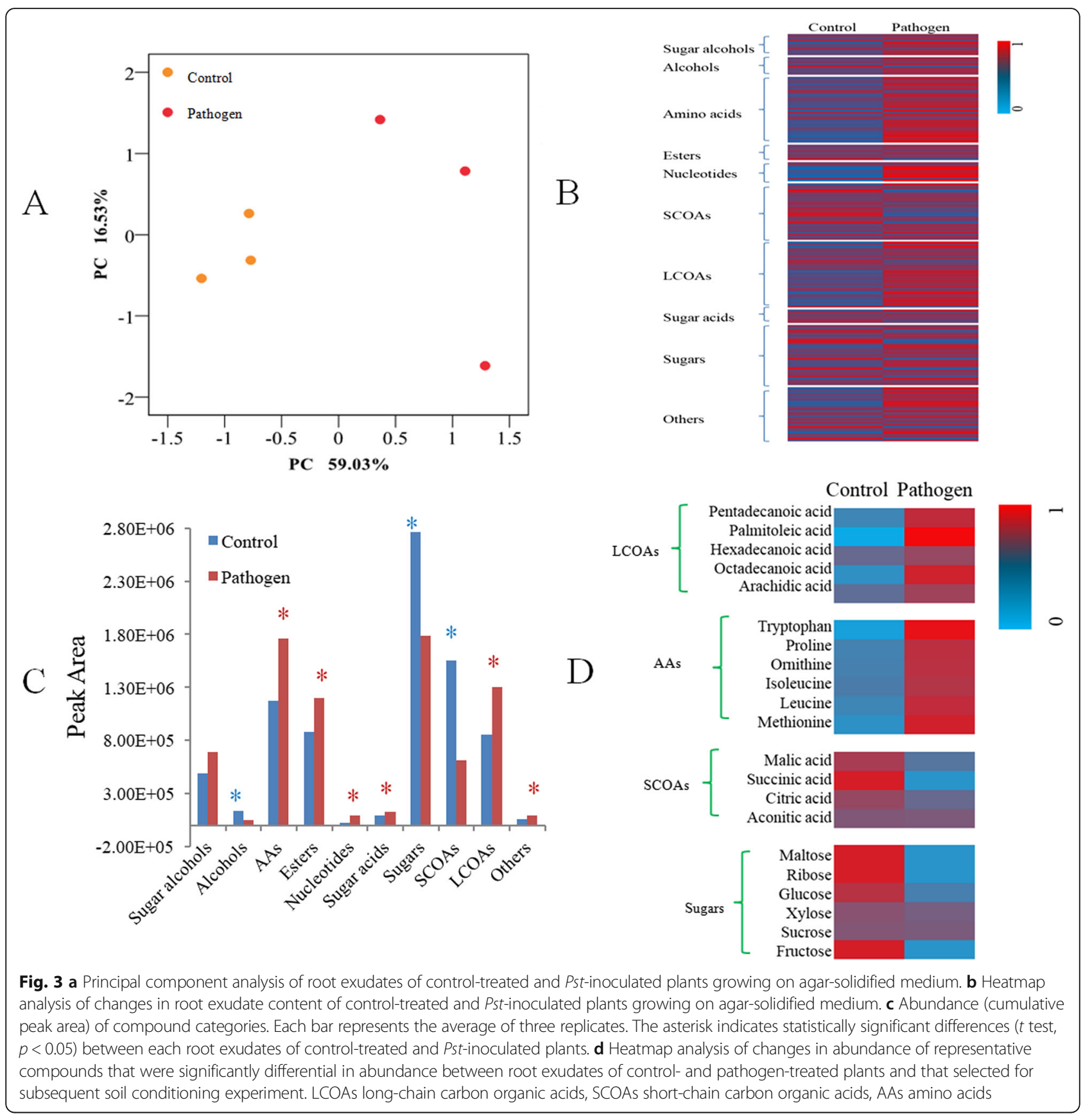

were still found to be significantly different according to PERMANOVA (Additional file 1: Table S3). This suggests that a large part of the microbial community contributes to the overall difference in community composition between the two soil compartments and two treatments. It is therefore difficult to pinpoint the extent to which specific ASVs, or combinations thereof, versus general community effects are responsible for the observed increase in plant resistance. However, it is clear that five generations of plants infected by Pst recruited a microbiome that was distinct from that of plants grown in the absence of the pathogen.

\section{Impact of Pst infection on root exudation profiles}

In order to establish a mechanistic explanation for how foliar infection of Arabidopsis alters the soil microbiome, root exudates of healthy and infected plants were collected in a gnotobiotic system and analyzed by gas chromatography-mass spectrometry (GC-MS). A total of 456 peaks were detected across all samples (Additional files 2). The overall exudation patterns from control plants were found to be distinct from those of plants infected with Pst, as demonstrated by their separation in a principal component analysis (PCA) $(p=0.043$ in ANOSIM, Fig. 3a). The 

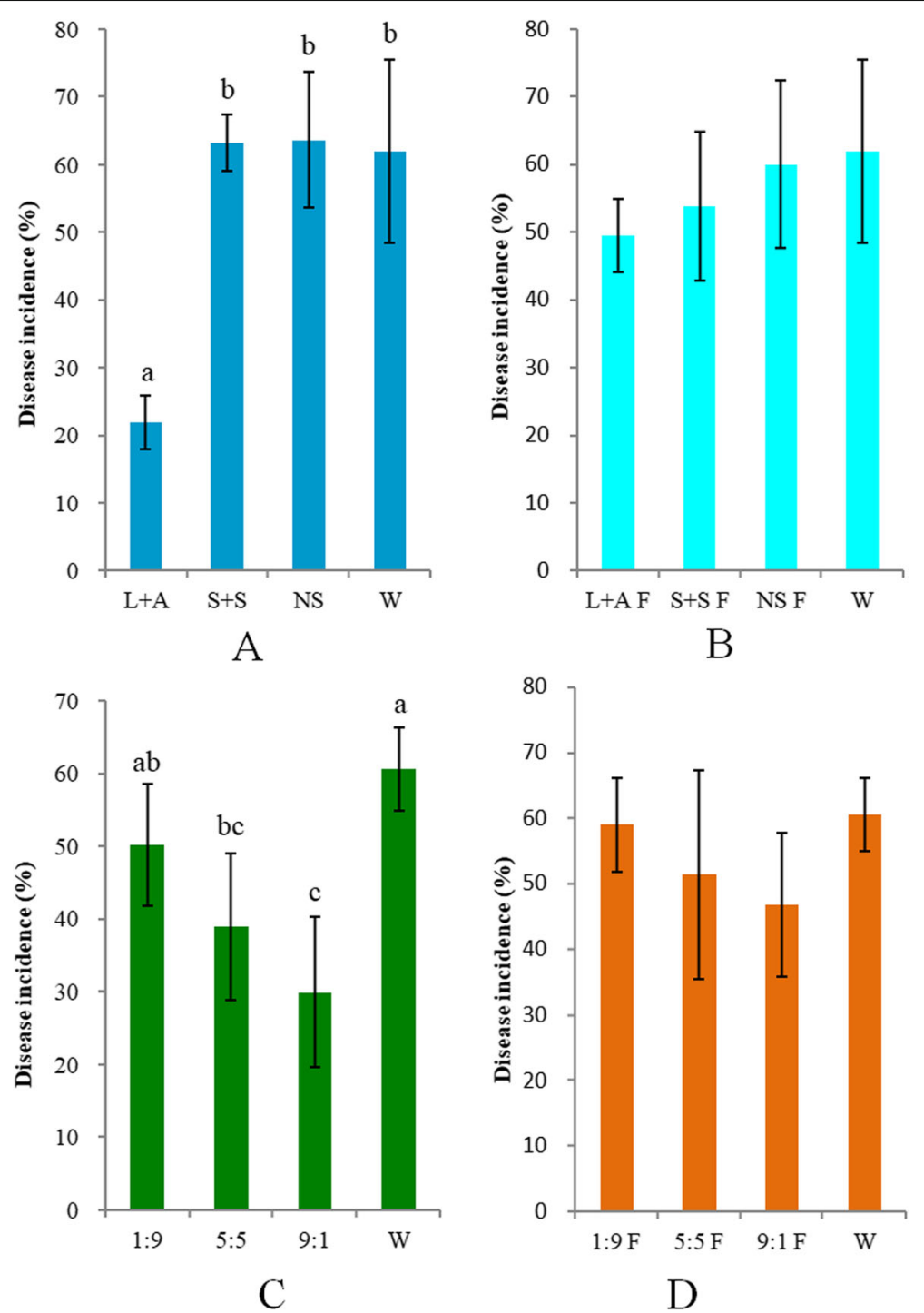

Fig. 4 a Disease incidence of Pst-challenged Arabidopsis plants growing on sterilized vermiculite-sand mixtures inoculated with water (W), slurries of natural soil (NS) or slurries of soils preconditioned with mixtures of LCOAs and AAs $(L+A)$, or SCOAs and sugars $(S+S)$. b Disease incidence of Pst-challenged Arabidopsis plants growing on sterilized vermiculite-sand mixtures inoculated with filter-sterilized W, NS, L + A or S + S. c Disease incidence of Pst-challenged Arabidopsis plants growing on sterilized vermiculite-sand mixtures inoculated with $W$ or $L+A$ and $S+S$ mixed in a ratio of 1:9, 5:5, or 9:1 ( $/ \mathrm{V})$. d Disease incidence of Pst-challenged Arabidopsis plants growing on sterilized vermiculite-sand mixtures inoculated with $W$ or filter sterilized $L+A$ and $S+S$ mixed in a ratio of 1:9, 5:5, or 9:1 $(v / V)$. Bars show average \pm SD of six replicates. Different letters indicate significant $(p<0.05)$ difference according to ANOVA with Tukey's post hoc test

compounds belonging to 201 of the 456 detected peaks could be identified and placed into broad categories based on their chemical nature, namely sugars (31 compounds), sugar acids (7), sugar alcohols (11), short-chain organic acids (29), long-chain organic acids (34), nucleotides (10), amino acids (34), esters (8), alcohols (9), or others (28). All of the identified compounds were detected in both treatments, but the abundance of 50 compounds differed significantly ( $t$ test, $p<0.05)$ between the two treatments (Fig. 3b). When evaluated at the group level, alcohols, short-chain organic acids (SCOAs), and sugars were found to be significantly lower ( $t$ test, $p<0.05)$ in the control, while esters, amino acids (AAs), nucleotides, sugar acids, and long-chain organic acids (LCOAs) were significantly higher in abundance in root exudates from infected plants (Fig. 3c). Overall, Pst infection resulted in a significantly higher secretion of long-chain carbon compounds while secretion of small compounds was reduced (Fig. 3c). LCOAs and AAs were more abundant in root exudates after pathogen infection, whereas SCOAs and sugars were less abundant (Fig. 3c). It has been suggested that simple sugar exudates serve as non-selective C substrates [40], 


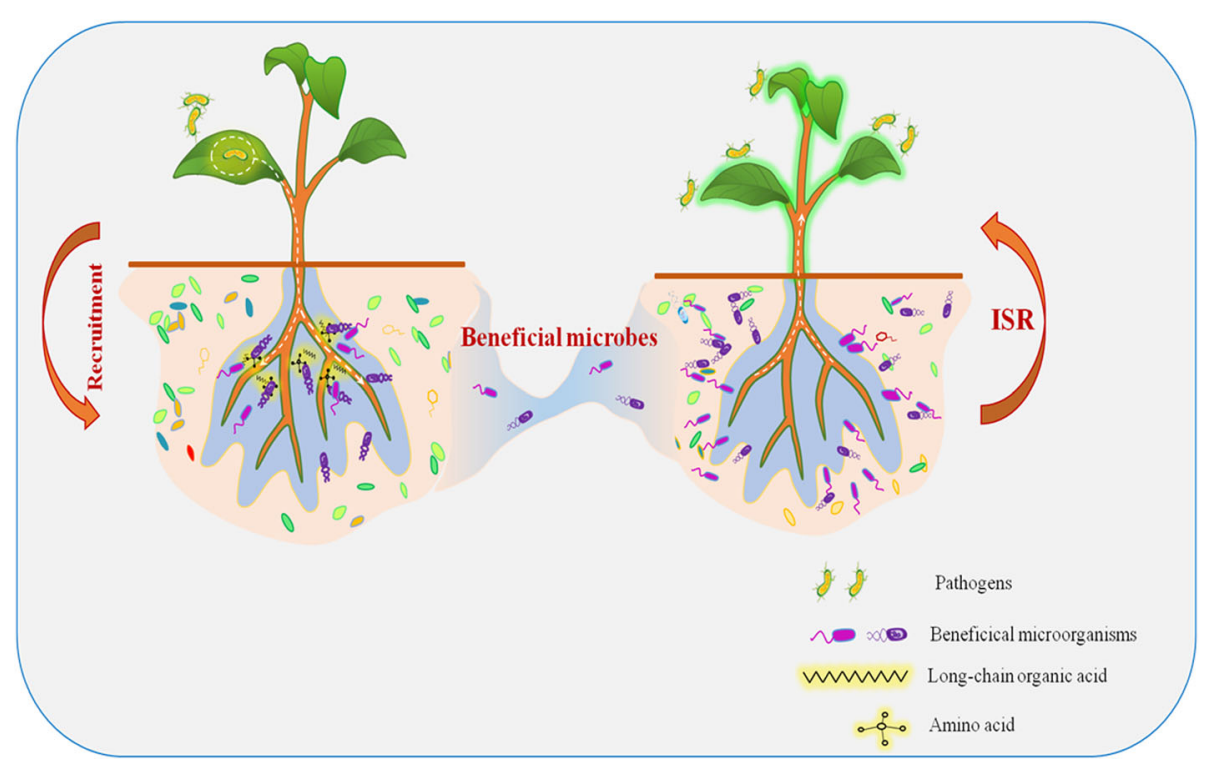

Fig. 5 Mechanistic model of soil-borne legacies induced by foliar pathogens. First, the predecessor plants release root exudates into soil to manipulate soil microbial community dynamics and recruit beneficial microbes when attacked by foliar pathogens. The resulting shifts then elicit phenotypic changes (such as phytohormone level) in the new plants to adapt to the pathogens' attack

while more complex organic acids exert more selective effects [41]. Indeed, previous studies have been shown that SCOAs, especially those involved in the tricarboxylic acid cycle, can recruit PGPRs in the rhizosphere [13, 42]. It is thus noteworthy that long-chain organic acids were detected at higher concentrations in root exudates of infected plants.

\section{Impact of exudation compounds on disease suppression}

To better understand the effect of differentially released compounds on soil microbes, we conditioned soil by repeatedly adding mixtures of compounds that were either over- or underrepresented in the exudation patterns of Pst-infected plants. To this end, exudate cocktails were made to represent four broad groups of differentially altered exudate compounds; LCOAs (pentadecanoic acid, hexadecanoic acid, palmitoleic acid, octadecanoic acid, and arachidic acid), the AAs (isoleucine, leucine, methionine, proline, tryptophan, and ornithine), SCOAs (citric acid, aconitic acid, succinic acid, and malic acid), and sugars (maltose, ribose, glucose, sucrose, fructose, and xylose) (Fig. 3d). Soil extracts were prepared from these pre-conditioned soils and used to inoculate naïve Arabidopsis plants grown in a sterilized mixture of sand and vermiculite, for subsequent challenge with Pst. The microbiome of soil preconditioned with mixtures of LCOAs and AAs $(\mathrm{L}+\mathrm{A})$ soils provided a greater level of induced resistance against foliar Pst than the microbiomes of control soils or soils pre-treated with SCOAs and sugars $(S+S)$ (Fig. 4a). Importantly, induction of disease resistance was lost in those soil extracts in which bacteria had been removed by filtration (Fig. 4b), indicating that the microbiomes, rather than exudation compounds themselves, were eliciting the resistance in Arabidopsis.

Soil transfer experiments were also preformed to assess the amount of microbiome addition needed to confer resistance, using different amounts $(10,50$, and $90 \%$ $w / w)$ of $\mathrm{L}+\mathrm{A}$ soil mixed into $\mathrm{S}+\mathrm{S}$ soil before soil slurry preparation. At least partial disease suppressiveness was conferred by the addition of $50 \%$ and $90 \%$ microbiome from $\mathrm{L}+\mathrm{A}$ soils to those of $\mathrm{S}+\mathrm{S}$ soils (Fig. 4c). Predictably, none of the filtered soil, and thus microbe-free, slurries were able to confer disease suppressiveness (Fig. 4d). Collectively, these results indicated that disease suppressiveness toward Pst was microbiologically induced in soils conditioned by $\mathrm{L}+\mathrm{A}$ mixtures.

\section{Conclusions}

In this study, we found that infections by the foliar pathogen Pst triggered a soil-borne legacy that induced resistance in a following generation of plants. This soilborne legacy was reflected by distinct bacterial communities in soils preconditioned by five generation of Pst-infected plants as compared to soils conditioned by non-inoculated plants. Moreover, when Arabidopsis thaliana was challenged by the foliar pathogen Pst, plant exudation patterns were altered and root secretions of LCOAs and AAs were increased. Application of a mixture of LCOAs and AAs to soil was sufficient to induce a similar soil microbiome-mediated pathogensuppressive response as observed after for actual response to the pathogen. 
Our combined results allow us to put forth a clear model related to responses to the aboveground pathogen (Fig. 5). We hypothesize that, upon infection, plant-systemic signaling leads to a change in root exudation profiles. These altered exudations in turn promote specific elements of the microbiome that induce resistance for future generations of the plant. Together, our results show not only that aboveground infection by the model bacterial pathogen Pst on the model plant Arabidopsis thaliana can have an effect on the resistance of subsequent plant populations growing in the same soil, but they also shed light on the mechanisms through which this soil-borne legacy is generated.

\section{Additional files}

Additional file 1: Figure S1. Schematic representation of the experimental design. Figure S2. Detection of Pseudomonas syringae pv. tomato strain DC3000 by PCR. The length of target fragment is 304 bp. Figure S3. Principal coordinate analysis (PCOA) with Bray-Curtis dissimilarity of the microbial community in bulk soil (B) or rhizosphere (R) of control (C) or pathogenconditioned (P) soils. A) bulk soils, B) rhizosphere soils. Figure S4. A and B are the relative abundance (\%) of the bacterial genera within the Firmicutes and Proteobacteria phyla in the microbial communities of control and pathogen- conditioned bulk soils, respectively. C: Relative abundance (\%) of the major bacterial phyla using the whole ASV table excluding ten differential ASVs (Fictibacillus and Sphingomonas) present in the microbial communities of control $(C)$ or pathogen-conditioned $(P)$ soils. Samples were taken from the bulk (B) soil or rhizospheres (R) of unchallenged plants. Figure S5. Principal coordinate analysis (PCoA) with Bray-Curtis dissimilarity of the microbial community in bulk soil (B) or rhizosphere (R) of control (C) or pathogenconditioned (P) soils using the whole ASV table excluding two ASVs belonging to the genera Fictibacillus and Sphingomonas, respectively. a) bulk soils; b) rhizosphere soils; c) all soil samples. Table S1. Soil properties in the control and pathogen-conditioned soil. Table S2. Relative abundance of two highly discriminative ASVs (Fictibacillus and Sphingomonas) in bulk soil samples from the control-conditioned (BC) versus pathogen-conditioned (BP) soils. Table S3. 200-300 ASVs together differentiate the microbial communities of pathogen conditioned and control bulks soil. (DOCX $690 \mathrm{~kb}$ )

Additional file 2: $\mathrm{R}$ code and root exudate compounds. (ZIP $360 \mathrm{~kb}$ )

\section{Acknowledgements}

We thank Ben Niu (Northeast Forestry University) for the comments on this manuscript.

\section{Funding}

This study was financially supported by China Science and Technology Ministry (973 Program, 2015CB150500). J. Y. was supported by the National Postdoctoral Program for Innovative Talents (BX201600075), Natural Science Foundation of Jiangsu Province (BK20170724), and National Natural Science Foundation of China (31330069).

\section{Availability of data and materials}

Sequence data are deposited in the NCBI Sequence Read Archive (SRA) database (SRP148928).

\section{Authors' contributions}

JY performed all experiments. JY and QS designed the study and wrote the majority of the manuscript. $\mathrm{RL}, \mathrm{QH}$, and JMV provided critical comments on the study and helped write the paper. JY, JZ, TW, PG, RLB, and MZ analyzed the data. RLB, YB, and GAK participated in the design of the study, provided comments, and edited the manuscript. All authors read and approved the final manuscript.
Ethics approval and consent to participate

Not applicable.

\section{Consent for publication}

Not applicable.

\section{Competing interests}

The authors declare that they have no competing interests.

\section{Publisher's Note}

Springer Nature remains neutral with regard to jurisdictional claims in published maps and institutional affiliations.

\section{Author details}

${ }^{1}$ Jiangsu Provincial Key Lab for Organic Solid Waste Utilization; National Engineering Research Center for Organic-based Fertilizers; Jiangsu Collaborative Innovation Center for Solid Organic Waste Resource Utilization, Nanjing Agricultural University, Nanjing 210095, China. ${ }^{2}$ School of Geography Science, Nanjing Normal University, Nanjing 210021, China. ${ }^{3}$ Plant-Microbe Interactions, Institute of Environmental Biology, Utrecht University, Padualaan 8, $3584 \mathrm{CH}$ Utrecht, the Netherlands. ${ }^{4}$ State Key Laboratory of Plant Genomics, Institute of Genetics and Developmental Biology, Chinese Academy of Science, Beijing 100101, China. ${ }^{5}$ Department of Horticulture and Landscape Architecture and Center for Rhizosphere Biology, Colorado State University, Fort Collins, CO 80523, USA. ${ }^{\circ}$ Ecology and Biodiversity Group, Department of Biology, Institute of Environmental Biology, Utrecht University, Padualaan 8, 3584 CH Utrecht, the Netherlands.

Received: 25 May 2018 Accepted: 23 August 2018

Published online: 12 September 2018

\section{References}

1. Weller DM, Raaijmakers JM, Gardener BB, Thomashow LS. Microbial populations responsible for specific soil suppressiveness to plant pathogens. Annu Rev Phytopathol. 2002;40:309-48.

2. Mazzola M. Assessment and management of soil microbial community structure for disease suppression 1. Annu Rev Phytopathol. 2004;42:35-59.

3. Rosenzweig N, Tiedje JM, Quensen JF, Meng Q, Hao JJ. Microbial communities associated with potato common scab-suppressive soil determined by pyrosequencing analyses. Plant Dis. 2011;96:718-25.

4. Cha J-Y, Han S, Hong H-J, Cho H, Kim D, Kwon Y, Kwon S-K, Crüsemann M, Lee YB, Kim JF. Microbial and biochemical basis of a Fusarium wiltsuppressive soil. ISME J. 2016;10(1):119-129.

5. Xiong W, Li R, Ren Y, Liu C, Zhao Q, Wu H, Jousset A, Shen Q. Distinct roles for soil fungal and bacterial communities associated with the suppression of vanilla Fusarium wilt disease. Soil Biol Biochem. 2017;107:198-207.

6. Mendes R, Kruijt M, de Bruijn I, Dekkers E, van der Voort M, Schneider JHM, Piceno YM, DeSantis TZ, Andersen GL, Bakker P, Raaijmakers JM. Deciphering the rhizosphere microbiome for disease-suppressive bacteria. Science. 2011; 332:1097-100

7. Pieterse CM, Zamioudis C, Berendsen RL, Weller DM, Van Wees SC, Bakker PA. Induced systemic resistance by beneficial microbes. Annu Rev Phytopathol. 2014:52:347-75

8. Berendsen R, Pieterse C, Bakker P. The rhizosphere microbiome and plant health. Trends Plant Sci. 2012:17:478-86.

9. Schlatter D, Kinkel L, Thomashow L, Weller D, Paulitz T. Disease suppressive soils: new insights from the soil microbiome. Phytopathology. 2017;107: 1284-97.

10. Kong HG, Kim BK, Song GC, Lee S, Ryu C-M. Aboveground whitefly infestation-mediated reshaping of the root microbiota. Front Microbiol. 2016;7:1314

11. Dudenhöffer JH, Scheu S, Jousset A. Systemic enrichment of antifungal traits in the rhizosphere microbiome after pathogen attack. J Ecol. 2016;104(6):1566-1575.

12. Berendsen RL, Vismans G, Yu K, Song $Y$, Jonge R, Burgman WP, Burmølle $M$, Herschend J, Bakker PAHM, Pieterse CMJ. Disease-induced assemblage of a plant-beneficial bacterial consortium. ISME J. 2018;12(6):1496-1507.

13. Rudrappa T, Czymmek KJ, Pare PW, Bais HP. Root-secreted malic acid recruits beneficial soil bacteria. Plant Physiol. 2008;148:1547-56.

14. Bakker PAHM, Pieterse CMJ, de Jonge R, Berendsen RL. The soil-borne legacy. Cell. 2018;172:1178-80. 
15. Raaijmakers JM, Mazzola M. Soil immune responses. Science. 2016;352:1392-3.

16. Badri DV, Chaparro JM, Zhang RF, Shen QR, Vivanco JM. Application of natural blends of phytochemicals derived from the root exudates of Arabidopsis to the soil reveal that phenolic-related compounds predominantly modulate the soil microbiome. J Biol Chem. 2013;288:4502-12.

17. Sasse J, Martinoia E, Northen T. Feed your friends: do plant exudates shape the root microbiome? Trends Plant Sci. 2018;23:25-41.

18. Gu Y, Wei Z, Wang X, Friman V-P, Huang J, Wang X, Mei X, Xu Y, Shen Q, Jousset A. Pathogen invasion indirectly changes the composition of soil microbiome via shifts in root exudation profile. Biol Fertil Soils. 2016;52: 997-1005.

19. Chaparro JM, Badri DV, Vivanco JM. Rhizosphere microbiome assemblage is affected by plant development. ISME J. 2014;8:790-803.

20. Li X, Bergelson J, Chapple C. The ARABIDOPSIS accession Pna-10 is a naturally occurring sng1 deletion mutant. Mol Plant. 2010;3:91-100,

21. Murashige T, Skoog F. A revised medium for rapid growth and bio assays with tobacco tissue cultures. Physiol Plant. 1962:15:473-97.

22. Yuan J, Chaparro JM, Manter DK, Zhang R, Vivanco JM, Shen Q. Roots from distinct plant developmental stages are capable of rapidly selecting their own microbiome without the influence of environmental and soil edaphic factors. Soil Biol Biochem. 2015;89:206-9.

23. Claesson MJ, O'Sullivan O, Wang Q, Nikkilä J, Marchesi JR, Smidt H, de Vos WM, Ross RP, O'Toole PW. Comparative analysis of pyrosequencing and a phylogenetic microarray for exploring microbial community structures in the human distal intestine. PLoS One. 2009;4:e6669.

24. Callahan BJ, McMurdie PJ, Rosen MJ, Han AW, Johnson AJA, Holmes SP. DADA2: high-resolution sample inference from Illumina amplicon data. Nat Methods. 2016;13:581.

25. Pruesse E, Quast C, Knittel K, Fuchs BM, Ludwig W, Peplies J, Glöckner FO. SILVA: a comprehensive online resource for quality checked and aligned ribosomal RNA sequence data compatible with ARB. Nucleic Acids Res. 2007;35:7188-96.

26. McMurdie PJ, Holmes S. Phyloseq: an R package for reproducible interactive analysis and graphics of microbiome census data. PLoS One. 2013;8:e61217.

27. Zhang Z, Esse HP, Damme M, Fradin EF, Liu CM, Thomma BPHJ. Ve1mediated resistance against Verticillium does not involve a hypersensitive response in Arabidopsis. Mol Plant Pathol. 2013;14:719-27.

28. Bakker MG, Chaparro JM, Manter DK, Vivanco JM. Impacts of bulk soil microbial community structure on rhizosphere microbiomes of Zea mays. Plant Soil. 2015;392:115-26.

29. Fiehn O, Wohlgemuth G, Scholz M, Kind T, Lee DY, Lu Y, Moon S, Nikolau B. Quality control for plant metabolomics: reporting MSI-compliant studies. Plant J. 2008;53:691-704.

30. Love Ml, Huber W, Anders S. Moderated estimation of fold change and dispersion for RNA-seq data with DESeq2. Genome Biol. 2014;15:550

31. Herms DA, Mattson WJ. The dilemma of plants: to grow or defend. Q Rev Biol. 1992:67:283-335.

32. Heil M, Hilpert A, Kaiser W, Linsenmair KE. Reduced growth and seed set following chemical induction of pathogen defence: does systemic acquired resistance (SAR) incur allocation costs? J Ecol. 2000;88:645-54.

33. Pieterse CM, Van dDD, Zamioudis C, Leon-Reyes A, Van Wees SC. Hormonal modulation of plant immunity. Annu Rev Cell Dev Biol. 2012;28:489.

34. Knight $\mathrm{H}$, Knight MR. Abiotic stress signalling pathways: specificity and cross-talk. Trends Plant Sci. 2001;6:262-7.

35. Pieterse CM, Leon-Reyes A, Van der Ent S, Van Wees SC. Networking by small-molecule hormones in plant immunity. Nat Chem Biol. 2009;5:308-16.

36. Vos IA, Verhage A, Schuurink RC, Watt LG, Pieterse CMJ, Van Wees SCM. Onset of herbivore-induced resistance in systemic tissue primed for jasmonate-dependent defenses is activated by abscisic acid. Front Plant Sci. 2013;4:539

37. Brouwer M, Lievens B, Van Hemelrijck W, Van den Ackerveken G, Cammue BP, Thomma BP. Quantification of disease progression of several microbial pathogens on Arabidopsis thaliana using real-time fluorescence PCR. FEMS Microbiol Lett. 2003:228:241-8.

38. Hiltner L. Über neuere Erfahrungen und Probleme auf dem Gebiete der Bodenbakteriologie unter besonderer Berücksichtigung der Gründüngung und Brache. Arbeiten der Deutschen Landwirtschaftlichen Gesellschaft. 1904:98:59-78

39. Kowalchuk GA, Buma DS, de Boer W, Klinkhamer PGL, van Veen JA. Effects of above-ground plant species composition and diversity on the diversity of soil-borne microorganisms. Antonie Van Leeuwenhoek. 2002;81:509.
40. Kamilova F, Kravchenko LV, Shaposhnikov Al, Azarova T, Makarova N Lugtenberg B. Organic acids, sugars, and L-tryptophane in exudates of vegetables growing on stonewool and their effects on activities of rhizosphere bacteria. Mol Plant-Microbe Interact. 2006;19:250-6.

41. Chaparro JM, Badri DV, Bakker MG, Sugiyama A, Manter DK, Vivanco JM. Root exudation of phytochemicals in Arabidopsis follows specific patterns that are developmentally programmed and correlate with soil microbial functions. PLoS One. 2013;8(2):e55731.

42. Yuan J, Zhang N, Huang Q, Raza W, Li R, Vivanco JM, Shen Q. Organic acids from root exudates of banana help root colonization of PGPR strain Bacillus amyloliquefaciens NJN-6. Sci Rep. 2015;5:13438.
Ready to submit your research? Choose BMC and benefit from:

- fast, convenient online submission

- thorough peer review by experienced researchers in your field

- rapid publication on acceptance

- support for research data, including large and complex data types

- gold Open Access which fosters wider collaboration and increased citations

- maximum visibility for your research: over $100 \mathrm{M}$ website views per year

At $\mathrm{BMC}$, research is always in progress.

Learn more biomedcentral.com/submissions 